\title{
UHF-RFID solutions for logistics units management in the food supply chain
}

\author{
Paolo Barge, Paolo Gay, Valentina Merlino, Cristina Tortia \\ DI.S.A.F.A - Università degli Studi di Torino, Grugliasco (TO), Italy
}

\begin{abstract}
The availability of systems for automatic and simultaneous identification of several items belonging to a logistics unit during production, warehousing and delivering can improve supply chain management and speed traceability controls. Radio frequency identification (RFID) is a powerful technique that potentially permits to reach this goal, but some aspects as, for instance, food product composition (e.g. moisture content, salt or sugar content) and some peculiarities of the production environment (high moisture, high/low temperatures, metallic structures) have prevented, so far, its application in food sector. In the food industry, composition and shape of items are much less regular than in other commodities sectors. In addition, a wide variety of packaging, composed by different materials, is employed. As material, size and shape of items to which the tag should be attached strongly influence the minimum power requested for tag functioning, performance improvements can be achieved only selecting suitable RF identifier for the specific combination of food product and packaging. When dealing with logistics units, the dynamic reading of a vast number of tags originates simultaneous broadcasting of signals (tag-to-tag collisions) that could affect reading rates and the overall reliability of the identification procedure. This paper reports the results of an extensive analysis of the reading performance of UHF RFID systems for multiple dynamic electronic identification of food packed products in controlled conditions. Products were considered singularly or arranged on a logistics pallet. The effects on reading rate and reading zone of different factors, among which the type of product, the number and position of antennas, the field polarization, the reader RF power output, the interrogation protocol configuration as well as the transit speed, the number of tags and their interactions were analysed and compared.
\end{abstract}

Correspondence: Cristina Tortia, DI.S.A.F.A, Università degli Studi di Torino, 44 Via Leonardo da Vinci, 10095 Grugliasco (T0), Italy

Tel. +39.011 .6708845 - Fax: +39.011 .2368845$

E-mail: cristina.tortia@unito.it

Key words: UHF, logistics, food supply chain.

Acknowledgements: this work was partially supported by the grants of the projects Namatech-Converging Technologies (CIPE2007), Regione Piemonte, Italy and PRIN 2009 (prot. 2009FXN7HW_002), MIUR, Italy.

(C) Copyright P. Bargeet al., 2013

Licensee PAGEPress, Italy

Journal of Agricultural Engineering 2013; XLIV(s2):e59

doi:10.4081/jae.2013.s2.e59

This article is distributed under the terms of the Creative Commons Attribution Noncommercial License (by-nc 3.0) which permits any noncommercial use, distribution, and reproduction in any medium, provided the original author(s) and source are credited.

\section{Introduction}

Traceability systems represent an important tool for companies to ensure the quality and safety of products, to increase the efficiency of managing of products and information flows and logistics, thus promoting their competitiveness within the supply chain and the market.

To cope with increasing complexity and globalization of supply chains, investments on ICT are constantly growing to improve information management and product identification systems efficiency. In particular, radio frequency identification (RFID) offers several advantages, as contactless and automatic identification of single or multiple products, which bestow an added value on this technology in comparison, for example, with barcodes. However, data transmission between the transponder and the reader takes place through electromagnetic waves and therefore without the need of free paths. Indeed, RFID tags are part of a technology that, when combined with databases or shared in communication networks, become an effective tool for the stakeholders involved in the supply chain, for new services and applications even in hostile environments. To reduce costs, for instance in logistics, transponders are usually inserted into labels to distinguish each unit of product of a specific production lot and to trace its position and path along the supply chain.

In the retail sector, RFID are commonly used for pallets, packing cases and/or single items identification to increase supply chain transparency, to reduce labour and optimize warehousing. Several important companies, e.g. Wal-Mart, Best Buy, Target and Albertson's, have already taken this opportunity. At receiving, RFID reduces labour, enabling the quick scanning of pallets and eliminating the need to open packing cases to get inventory down to item level. Benefits include reducing out-of-stocks through item-level visibility of storeroom and shelf inventories, reducing the mis-shipments by promoting more accurate shipping, and preventing product counterfeiting, diversions and theft through mass, reliable, item-level identification (Penttilä et al., 2006).

RFID systems operating at UHF band can be used to improve single items, automatic, simultaneous and contactless data capture. RFID can be applied at different level as wholesale distributors for automatic inventory management, at partner/supplier depots, on the loading dock for automatic identification of pallet. In food supply chains some issues remain to be addressed for the application of this technology. These include reading reliability, which in some cases and with certain products is still to be improved, the lack of homogeneity of the standards governing their use in different countries at the global level, the investment costs and the need to involve stakeholders and upstream and downstream. For these reasons, although this represents a potential solution for goods traceability and for supply chain logistics control, the introduction of RFID UHF technology in different food sectors is currently being researched. There are some noticeable technical difficulties to obtain exhaustive and reliable identification of food items in critical production environments (Clarke et al., 2006; Mühlmann and Witschnig, 2007). The main responsible of the negative effects on tag performances, both in static and dynamic condi- 
tions, is the variation of the electric field in the proximity of a food object due to its dielectric properties which influence reflections, transmission and absorption rates. It is therefore important to assess food behaviour in processes where electromagnetic fields are involved, in order to avoid scarce reliability of the system that could definitively lead to the loss of confidence by the end user.

Collisions among tags in a dense population conditions is another critical aspect.

The EPCglobal Gen2 protocol regulates the interaction between RFID reader and tags operating in the $860 \mathrm{MHz}-960 \mathrm{MHz}$ frequency range defining the physical and logical requirements for a passive-backscatter, interrogator-talks-first system. This standard is widely used for supply chain management where medium-range and high-speed identification is required (Wen-Tzu and Wen-Bin, 2011). In EPC Gen2 protocol, which is nowadays the widespread adopted UHF standard, interrogation phase is controlled adjusting some key-parameters that influence the duration and the accuracy of a complete tag inventory. The tuning of these parameters is crucial in dynamic applications, as the collisions must be quickly solved, while the objects to be identified are still in the reading area.

To read multiple tags simultaneously in a dense population, an anticollision algorithm (Q), based on dynamic framed slotted ALOHA method, was developed. As soon as the tag enters the RF field domain, is enabled to accept the interrogator request. A selection of a group of tags can be made by a query command which communicate to the tag one among four sessions: S0, S1, S2 and S3. Each session is provided with a two-state inventoried flag (A or B). When the tag is energized, the persistence time of the inventoried flag value is indefinite for S0, $\mathrm{S} 2$ and $\mathrm{S} 3$, while is in the range $\mathbf{0 . 5}-5 \mathrm{~s}$ for $\mathrm{S} 1$ session. The algorithm implements a number of slotted frames equal to $2^{\mathrm{Q}}-1$. Each of the selected tags randomly choose one among the available time slot (in the range $0,2^{\mathrm{Q}}-1$ ) and will backscatter a 16 -bit random number (RN16) when the interrogator calls for the specified time slot. Upon receiving a tag RN16, the interrogator acknowledges the tag with the same RN16. If the RN16 is correct, the tag will finally backscatter its 96-bits ID. At this point, the tag commutates to the acknowledged state and also inverts the Inventoried flag specified by the 'Query' command.

In this paper, technological solutions for packed food products automatic and simultaneous identification based on RFID-UHF system were reported and discussed. The system is intended to improve the internal logistics (identification, localization, automatic count, inventory management) and through the supply chain, as well as to allow the automatic collection of production process information. A very diffused RFID solution at industrial level is the portal (or gate) layout, which is generally implemented across conveyor belts or in loading dock area for the automatic loading/unloading of pallet in warehouses. At this purpose a RFID gate, constituted of a metallic structure and equipped with $4 \mathrm{UHF}$ antennas, was constructed to perform simultaneous reading of a large number of tags attached on packed food arranged on a pallet. Different kinds of food materials were used for trials: dry food, beverage and their respective empty packaging were selected to compose the pallet for electronic identification. Different technological solutions were evaluated in terms of reading accuracy of tags applied on secondary packaging, considering static and dynamic reading conditions and different antenna layout.

\section{Materials and Methods}

To evaluate the performances of UHF identification of food packed products, an experimentation on items housed in secondary packaging was carried out at first in static conditions.

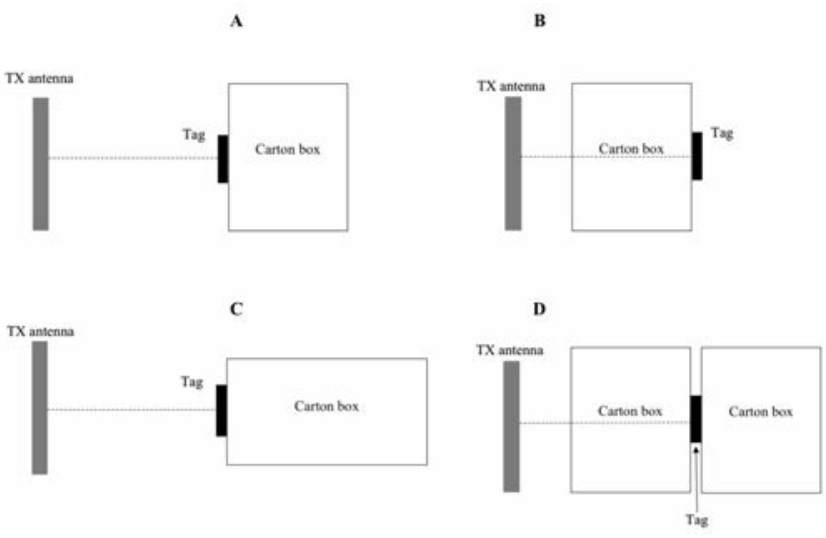

Figure 1. Position of food items, transmitting and receiving antennas in the four configurations $\mathrm{A}, \mathrm{B}, \mathrm{C}$, and $\mathrm{D}$.

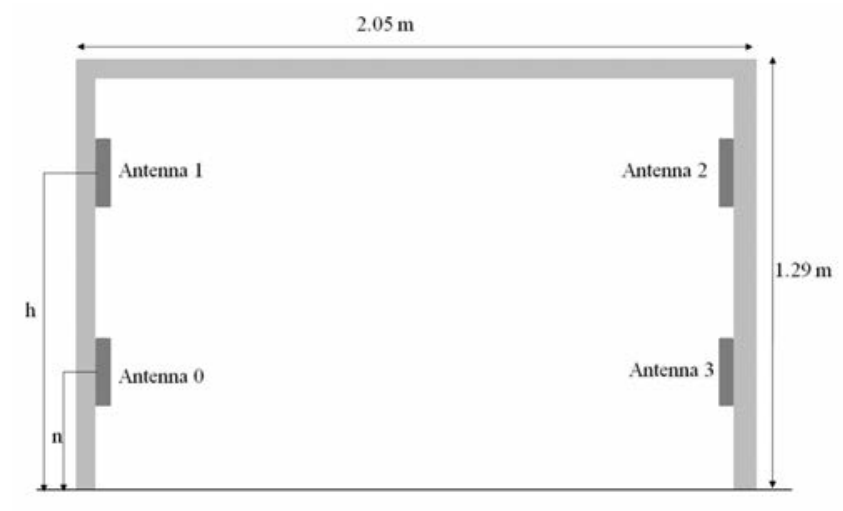

Figure 2. Gate framework: dimensions and antennas position.
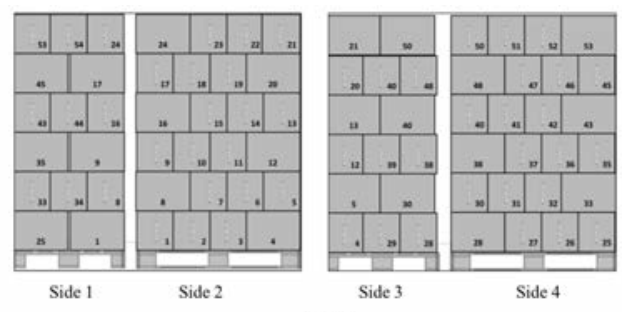

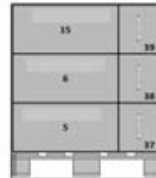

Side 1

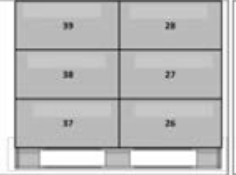

Side 2

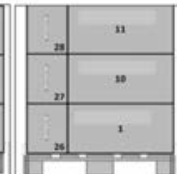

Side 3

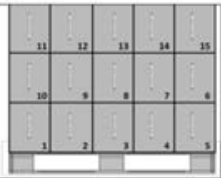

Side 4
Figure 3. Snacks and empty boxes $(\mathrm{Y})$ and sweetened tea $(\mathrm{Z})$ pallet setup. 
At this purpose, tag interrogation was carried out using a Caen RFID R4300P standalone reader connected to a linear (Caen RFID, model Wantenna X007, $8 \mathrm{dBi}$ gain) or to a circular polarized antenna (Caen RFID, model Wantenna X005, $7 \mathrm{dBi}$ gain). The system is IS0 18000-6C compliant. The effect of the packed food on minimum transmitter power output (TP0) to activate the tag and correctly receive the EPC ID was evaluated measuring the parameter $P_{\min }(\mathrm{dB})$, as described by Rao et al. (2005) and Nikitin et al. (2007). $P_{\min }$ was determined in semi anechoic chamber, at a fixed distance, modulating TP0 by a power sweep in the range $0 \div 2000 \mathrm{~mW}$ till tag detection (Tortia et al., 2012).

Five tags, LabID UH100 (a), LabID UH3D40 (b), LabID UH331 (c), Alien $9634(d)$, and Alien $9662(e)$, were tested in free space or directly attached on the secondary packaging of the food products. Firstly, to compare transponders performances, the minimum power to activate each of the considered tag models was measured with the transponder attached to a very thin plastic tape suspended in free space by means of a light wood frame. The tag-to-reader antenna distance was maintained constant at $0.5 \mathrm{~m}$. Both circular and linear polarized antennas were used for tests. UHF attenuators $(-10 \mathrm{dBm}$ for circular polarized antenna and $-20 \mathrm{dBm}$ for linear polarized antenna) were used to maintain the reader in the nominal output power range.

In a second trial, tags were attached directly on a carton box filled with sweet snacks or sweetened tea in four configurations to simulate possible item positioning scenarios (Figure 1). Sweet snacks were singularly packed by a carton/polypropylene film primary packaging; 120 single items were then inserted in a secondary carton box $(0.3 \times 0.4 \mathrm{x}$ $0.25 \mathrm{~m}$ ). Sweetened tea was contained in plastic cups welded at the top in group of six items and secondarily packed stacking 72 single items in a carton box $(0.28 \times 0.56 \times 0.22 \mathrm{~m})$. A single electronically identified carton box in absence (configurations A, B, and C) or presence (D) of another identical item in proximity was adopted in this trial. In configuration B and D the product is interposed between reader (TX) and tag (RX) antennas, while only free space separates RX and TX antenna in $\mathrm{A}$ and $\mathrm{C}$ configurations. Tag-to-reader antenna distance was maintained constant at $1 \mathrm{~m}$.

This approach allows assessing the power levels to be employed by the reader antenna to identify food materials with different dielectric properties. Then, dynamic reading efficiency (DRE \% - tag correctly identified/tag present) was evaluated simulating identification in dynamic condition, i.e. with the product moving through a portal, using a UHF gate with several of the previous product items arranged on a moving pallet.

The evaluation of the performances of the UHF systems in dynamic conditions was carried out employing a portal where four UHF antennas were mounted. In Figure 2 the portal framework with the layout of the four antennas (named $0,1,2$, and 3 ) is reported.

No metals were present in the working environment as the gate was in a large empty room. The major contribution to reflection, apart of the gate metal framework, is due to the floor (Sydänheimo et al., 2006). Caen A941 standalone reader controlled by the RFID Caen Easy Controller software was used. To compare performances in dynamic conditions, the variable tag was excluded, using only $\operatorname{tag} c$. Each carton box was arranged on a pallet following the scheme in Figure 3 and then electronically identified. Tags were always attached on the external minor side of the carton box. Pallet $Y(1.80 \times 1.15 \times 1.20 \mathrm{~m})$ was composed of 54 carton boxes arranged in six horizontal rows of nine carton boxes for each level. Carton boxes in this pallet were empty or filled with sweet snacks for a total of 648 primary packed items. Pallet $Z(0.84$ $\mathrm{x} 1.10 \times 0.78 \mathrm{~m}$ ) was composed by three horizontal rows and seven carton boxes filled with sweetened tea per each level for a total of 21 carton boxes.

Identification of empty boxes pallet was used to verify the reading coverage of the gate at different combinations of linear and circular antennas. Firstly, two linear ( $n=0.73 \mathrm{~m}$ - antennas 0 and 3$)$ and two circular ( $h=1.34 \mathrm{~m}$ - antennas 1 and 2) polarized antennas were mounted on the gate. TPO was set to $1000 \mathrm{~mW}$. In dynamic identification trials pallet was manually moved through the gate using a forklift at different speeds ranging from 0.5 to $1.5 \mathrm{~ms}^{-1}$. Pallet was always forked from the narrow side: in this way, the pallet larger side was parallel to the antennas plane. On the contrary, for the static tests, pallet was positioned under the RFID portal until the end of the reading cycle (10 seconds).

In the following, four linear polarized antennas were used both for dynamic and static readings using both $Y$ configuration with carton boxes empty or filled with sweet snacks and $Z$ configuration for tea.

Table 1. Configurations of the four linear polarized antennas in the gate. $\mathrm{n}$ is the height of antennas $\mathbf{0}$ and 3 , while $\mathrm{h}$ is the height of antennas 1 and 2. Horizontal shift is the distance from the antenna centre to the gate vertical central axis. Elevation angle is the angle between the antenna and the horizontal plane.

\begin{tabular}{lcccc} 
Configurations & $\begin{array}{c}\text { Height } \\
(\mathrm{m})\end{array}$ & $\begin{array}{c}\text { Antennas } \\
\text { Horizontal shift } \\
(\mathrm{m})\end{array}$ & $\begin{array}{c}\text { Blevation } \\
\text { angle }\end{array}$ \\
& $\mathrm{n}$ & 0.73 & 0 & $90^{\circ}$ \\
& $\mathrm{h}$ & 1.48 & & \\
2 & $\mathrm{n}$ & 0.25 & +0.16 & $90^{\circ}$ \\
& $\mathrm{h}$ & 1.48 & 0 & $110^{\circ}$ \\
\hline 3 & $\mathrm{n}$ & 0.44 & +0.16 & $90^{\circ}$ \\
& $\mathrm{h}$ & 1.35 & 0 & $90^{\circ}$ \\
4 & $\mathrm{n}$ & 0.44 & $0.16 /-0.16$ & $100^{\circ}$ \\
& $\mathrm{h}$ & 1.35 & $-0.16 / 0.16$ & $75^{\circ}$ \\
\hline 5 & $\mathrm{n}$ & 0.44 & $-0.16 / 0.16$ & $100^{\circ}$ \\
& $\mathrm{h}$ & 1.15 & $0.16 /-0.16$ & $90^{\circ}$ \\
& $\mathrm{n}$ & 0.65 & $0 * 0$ & $90^{\circ}$ \\
& $\mathrm{h}$ & 1.46 & & \\
\hline
\end{tabular}

Table 2. Mean $P_{\min }$ of each tag model attached on a thin plastic tape suspended in free space by means of a light wood frame. Tag to reader antennas distance was equal to $0.5 \mathrm{~m}$.

\begin{tabular}{lccc} 
Tag & Model & $\begin{array}{c}\text { Circular } \\
\text { polarization } \\
\text { antenna }\end{array}$ & $\begin{array}{c}\text { Polarization } \\
\text { antenna }\end{array}$ \\
\hline a & UH100 & 10.7 & 2.0 \\
b & UH3D40 & 12.3 & 9.5 \\
\hline c & UH331 & 9.8 & 2.1 \\
d & ALIEN9634 & 10.1 & 1.3 \\
\hline e & ALIEN9662 & 11.0 & 2.4 \\
\hline
\end{tabular}

Table 3. Pmin (dBm) to activate tag $\mathrm{c}$ by CAEN linear polarized antenna. Tag was attached on carton box filled with sweet snacks or plastic cups of sweetened tea in different reading configurations. Tag to antenna distance was equal to $1 \mathrm{~m}$.

\begin{tabular}{lcc} 
Configurations & \multicolumn{2}{c}{ Pmin } \\
A & Sweet dry Snack & Sweetened tea \\
B & 17.2 & 22.8 \\
\hline C & 17.4 & Out of range \\
D & 20.7 & 24.1 \\
\hline
\end{tabular}


Table 4. Dynamic reading efficiency $\left(\mathrm{DRE} \%=\mathrm{n}^{\circ}\right.$ tags correctly identified $/ \mathrm{n}^{\circ}$ tags present) of electronic identification of pallets $\mathrm{Y}$ and $\mathrm{Z}$, also with empty carton boxes, by means of four linear polarized antennas, adjusted in different configurations, in static and dynamic conditions in functions of $Q$ and $S$ values and, in the case of dynamic conditions, of pallet speed through the gate.

\begin{tabular}{|c|c|c|c|c|c|c|}
\hline Carton box & Q & S & $\begin{array}{c}\text { Static or dynamic } \\
\text { (S/D) }\end{array}$ & $\begin{array}{l}\text { Speed } \\
(\mathrm{m} / \mathrm{s})\end{array}$ & $\begin{array}{c}\text { Antennas } \\
\text { configuration }\end{array}$ & DRE\% \\
\hline \multirow[t]{10}{*}{ Empty } & 1 & 2 & S & 0 & 1 & 96 \\
\hline & 1 & 2 & D & 0.80 & & 94 \\
\hline & 2 & 2 & S & 0 & 1 & 100 \\
\hline & 2 & 2 & D & $0.55 \div 0.80$ & & 100 \\
\hline & 2 & 2 & D & $0.99 \div 1.21$ & & 90 \\
\hline & 3 & 2 & S & 0 & 1 & 100 \\
\hline & 3 & 2 & D & 0.53 & & 100 \\
\hline & 3 & 2 & D & $0.57 \div 0.60$ & & 98 \\
\hline & 3 & 1 & S & 0 & 1 & 100 \\
\hline & 3 & 1 & D & $0.40 \div 0.70$ & & 94 \\
\hline \multirow[t]{6}{*}{ Dry Snack } & 2 & 2 & S & 0 & 3 & 100 \\
\hline & 2 & 2 & D & $0.50 \div 0.70$ & & 100 \\
\hline & 3 & 2 & S & 0 & 1 & 96 \\
\hline & 3 & 2 & S & 0 & 2 & 100 \\
\hline & 3 & 2 & D & $0.50 \div 0.60$ & & 100 \\
\hline & 3 & 2 & D & 1.24 & & 88 \\
\hline \multirow[t]{3}{*}{ Sweetened tea } & 2 & 2 & S & 0 & 4 & 80 \\
\hline & 2 & 2 & S & 0 & 5 & 100 \\
\hline & 2 & 2 & $\mathrm{D}$ & $0.30 \div 0.50$ & & 100 \\
\hline
\end{tabular}

Several configurations with variable antennas heights, positions and orientations were tested (see Table 1 for details) to verify the coverage zone and reading efficiency of the gate.

Four linear polarized antennas were fixed on the portal in configuration 6 (Table 1). The adopted power level ranged from $200 \mathrm{~mW}$ to 800 $\mathrm{mW}$, while the forklift speed through the gate was ranging from 0.5 to $2 \mathrm{~ms}^{-1}$. Electronic identification of pallets $Y$ was performed also rotating the external carton boxes in order to position the tag on the internal side of the pallet.

Both in static and dynamic trials, Session (S0, S1, S2, and S3) and Q values were changed in the different repetitions to evaluate the influence of these parameters of the ALOHA anti-collision algorithm on reading efficiency.

\section{Results}

Minimum power levels to be delivered from the reader to obtain tag activation at $0.5 \mathrm{~m}$ distance are reported, for each tag model and receiving antenna combination, in Table 2 . As we refer to the power at the cable before the antenna panel, Equivalent Isotropic Radiated Power $(E I R P)$ in the tag direction is different for antennas with higher/lower gain. Since to detect the tag the electromagnetic signal has to cover both forward and backward paths, the transmitter and tag antenna gains influence twice the required power levels.

As for passive tag the power received at the reader goes as the inverse fourth power of the distance between reader and tag antennas, and it is also proportional to the square of antenna gain, this latter plays a large role in achievable read range. For this reason, $P_{\text {min }}$ mean values (TPO at the cable, $\mathrm{dBm}$ ) are different, even for the same tag model, when using circular or linear antennas.

The comparison among tags was done ranking results obtained with the same antenna. As can be seen, the activation of $\operatorname{tag} b$ is critical and higher power levels are needed in both linear and circular polarization cases (Table 2), even if in the case of circularly polarized field the difference with other tags is less evident. This could be explained consid-
Table 5. Dynamic reading efficiency (DRE\%) at different power levels and pallet speeds. Portal was in configuration 6 and both snacks $(Y)$ and tea pallets $(\mathrm{Z})$ were used.

\begin{tabular}{lccccc} 
Pallet & $\begin{array}{c}\text { Repetitions } \\
(\mathrm{n})\end{array}$ & $\begin{array}{c}\text { TPO } \\
(\mathrm{mW})\end{array}$ & $\begin{array}{c}\text { Speed range } \\
(\mathrm{m} / \mathrm{s})\end{array}$ & $\begin{array}{c}\text { Medium speed } \\
(\mathrm{m} / \mathrm{s})\end{array}$ & DRE\% \\
$\mathrm{Y}$ & 15 & 200 & $1.30-2.10$ & 1.45 & 100 \\
$\mathrm{Z}$ & 4 & 100 & $0.50-0.90$ & 0.73 & 84.75 \\
\hline $\mathrm{Z}$ & 11 & 200 & $0.50-1.50$ & 1.08 & 97.8 \\
$\mathrm{Z}$ & 2 & 400 & $0.11-0.9$ & 0.51 & 100 \\
\hline $\mathrm{Z}$ & 3 & 800 & $0.9-1.4$ & 1.10 & 100 \\
\hline
\end{tabular}

ering that tag $b$ is a double dipole cross-shaped which can receive power at different field polarization orientations.

The power requested to activate tag $d$ resulted low, but from our previous experiences (Barge et al., 2013), performances of this transponder type are strongly reduced when attached to food products. On the basis of these results, $\operatorname{tag} c$ was chosen for static performances evaluation on food items contained in their secondary packaging both in static and dynamic conditions.

Table 3 reports minimum power levels to activate transponder $c$ attached to the carton box filled with snacks or tea in different configurations. The correct identification of the tag was always possible at $1 \mathrm{~m}$ distance for carton filled with snacks, even when the packed product was interposed between reader and tag antennas (B and D). It can be noticed that, for snacks, the positioning of the tag on the minor side, leaving a thicker mass behind the tag in length (C), has a stronger effect on the required power than the interposition of the object in front of the tag (A). For sweetened tea, tag reading was possible only if tea was not interposed among tag and reader (B and D).

In dynamic trials, when the gate was composed by a combination of two linear and two circular polarized antennas and empty carton boxes were stacked on the pallet following scheme $Y$, mean $D R E \%$ was not acceptable (93\%), even at low speed $\left(<0.3 \mathrm{~ms}^{-1}\right)$ and with session set to $\mathrm{S} 0$. It has to be reminded that, at constant $Q$ value, the probability to have collisions increases if in the field are present a larger number of 
tags matching the chosen flag value. In case of session S0, when the tag has already transmitted its EPC code the inventoried flag changes value (e.g. from A to B) till tag is energized, but if tag is not powered, even if for a very short period, it returns to the default value (e.g. A) thus participating again to the group of tags not yet inventoried.

In dynamic conditions, the probability that tag loses its power, is very high and thus, if session is S0, as the group of tags to be selected is always large, collisions are increased.

In static conditions, when tags are continuously energized, all the transponders were correctly detected.

As the low gain of circular polarization antenna resulted in a more difficult detection of the tags, only linear polarized antennas were considered in the following trials. Circular polarization antennas, however, must be considered in case of misalignment between field orientation and tag.

Different session and Q values combinations were then used, as reported in Table 4.

When $Q$ value was set to 1 only two slotted frame are available for tags to send their RN16 code. As the tag number is 54, collisions are very frequent and the reading is difficult even in static condition. In fact, if $Q$ was set to 2 and 3 in static conditions the complete reading of tag present in the field for empty boxes was always obtained. The $Q$ value must be well adapted to the number of expected tag as the bigger the number of slot, the longer is the reading cycle to acknowledge all tag IDs. As in case of $Q$ set to 3 was not observed a great improvement of DRE, for sake of reading speed, $Q$ value 2 should be preferred. If session was set to $\mathrm{S} 1$ even on empty boxes and at low speed the complete reading of tag was not achieved. This is probably due to the inventoried flag persistence time which is in the range $0.5-5 \mathrm{~s}$, even if the tag is in energized state. In fact, when working with session 0 and 1 , each tag code was acquired more than two times per inventory round. As configuration 1 was not optimal when in cartons food products were present, antenna position was changed (configurations 2-5). In particular antenna height was changed as floor acted as reflector, and horizontal position was shifted to increase reading volume.

In configuration 2 and 3 the DRE was $100 \%$ at speed up to $0.6 \mathrm{~ms}^{-1}$ for sweetened snacks.

Reading results for tea were better than expected as dynamic reading of all the 21 tags was possible, even if the speed should be maintained up to $0.5 \mathrm{~ms}^{-1}$.

In Table 5 is highlighted the effect of increasing TPO on the reading efficiency for tea, at different speed, in configuration 6 , which resulted to be optimal for each kind of tested product.

In this configuration, for snacks, some of the boxes of pallet $Y$ were turned and the tag remained internal to other boxes. Also in this critical tag position a $100 \%$ DRE was obtained.

\section{Conclusions}

The influence of tag positioning on food products was assessed comparing two food types, characterized by different dielectric properties as well as their empty packaging.

Dynamic efficiency of RFID UHF portal were compared in various antennas geometries, TPO levels and EPC Gen2 protocol parameters tuning using three pallet setup assembling both empty, dry snacks and sweetened tea carton boxes.
In the case studied, where tag were all optimally oriented for linear polarized antennas, the circular polarization antenna resulted less efficient in dynamic conditions.

The dynamic reading efficiency as well as the time to complete the inventory are strongly dependent on anti-collision algorithm parameters. The parameter $Q$ must be optimized in function of the number of the expected tag and the speed of object moving through the portal. The persistence of the inventoried flag of the tag, which is typical for different sessions, is relevant to limit collision during inventory, increasing accuracy.

As often, at shipping, different products are picked and a heterogeneous pallet composition is obtained, if RFID is used as tracking device, the scheme used for positioning of the packed products must be optimized in function of the discussed results.

\section{References}

Barge, P., Gay, P., Merlino, V., Tortia, C., 2013. Effect of packaging on Radio Frequency Identification of food products. From effective to intelligent agriculture and forestry, XXXV CIOSTA \& CIGR V Conference 2013, 3 - 5 July, Billund, Denmark. In press.

Clarke, R.H., Twede, D., Tazelaar, J.R., Boyer, K.K., 2006. Radio frequency identification (RFID) performance: the effect of tag orientation and package contents. Packaging Technology and Science $19,45-54$.

EPCGlobal Inc ${ }^{\mathrm{TM}}$, 2005. EPC ${ }^{\mathrm{TM}}$ Radio-Frequency Identity Protocols Class-1 Generation - 2 UHF RFID - Protocol for communications at $860 \mathrm{MHz}-960 \mathrm{MHz}$. Version 1.0.9.

Mühlmann, U., Witschnig, H., 2007. Hard to read tags: an applicationspecific experimental study in passive UHF RFID systems. Elektrotechnik und Informationstechnik, 124(11), 391396.

Nikitin, P.V., Rao, K.V.S., Lazar, S., 2007. An Overview of Near Field UHF RFID, in: IEEE International Conference on RFID, 2007. Presented at the IEEE International Conference on RFID, 2007, $167-174$.

Penttilä, K., Keskilammi, M., Sydänheimo, L., Kivikoski, M., 2006. Radio frequency technology for automated manufacturing and logistics control. Part 2: RFID antenna utilisation in industrial applications. The International Journal of Advanced Manufacturing Technology 31, 116-124.

Rao, K.V.S., Nikitin, P.V., Lam, S.F., 2005. Antenna design for UHF RFID tags: a review and a practical application. IEEE Transactions on Antennas and Propagation 53, 3870 - 3876 .

Sydänheimo, L., Ukkonen, L., Kivikoski, M., 2006. Effects of size and shape of metallic objects on performance of passive radio frequency identification. The International Journal of Advanced Manufacturing Technology 30, 897-905.

Tortia, C., Barge, P., Gay, P., Merlino, V., Serale, S. and C. Gandini, 2012. Key technological factors for successful RFID systems application in food supply chain management. CIGR-AGENG 2012, International Conference of Agricultural Engineering "Agriculture and Engineering for Healthier Life", July 8-12, Valencia, Spain.

Wen-Tzu, C., Wen-Bin, K., 2011. A Novel Q-algorithm for EPCglobal Class-1 Generation-2 Anti-collision Protocol. World Academy of Science, Engineering and Technology 54, 801-804. 